\title{
Article \\ Unveiling the Intramolecular Ionic Diels-Alder Reactions within Molecular Electron Density Theory
}

\author{
Luis R. Domingo *(D), Mar Ríos-Gutiérrez (D) and María José Aurell (D) \\ Department of Organic Chemistry, University of Valencia, Dr. Moliner 50, Burjassot, E-46100 Valencia, Spain; \\ rios@utopia.iv.es (M.R.-G.); maria.j.aurell@uv.es (M.J.A.) \\ * Correspondence: domingo@utopia.uv.es
}

check for updates

Citation: Domingo, L.R.;

Ríos-Gutiérrez, M.; Aurell, M.J. Unveiling the Intramolecular Ionic Diels-Alder Reactions within Molecular Electron Density Theory. Chemistry 2021, 3, 834-853. https:/ / doi.org/10.3390/chemistry3030061

Academic Editor: Roberto Cammi

Received: 23 June 2021

Accepted: 26 July 2021

Published: 3 August 2021

Publisher's Note: MDPI stays neutral with regard to jurisdictional claims in published maps and institutional affiliations.

Copyright: (c) 2021 by the authors. Licensee MDPI, Basel, Switzerland. This article is an open access article distributed under the terms and conditions of the Creative Commons Attribution (CC BY) license (https:// creativecommons.org/licenses/by/ $4.0 /)$.
Abstract: The intramolecular ionic Diels-Alder (IIDA) reactions of two dieniminiums were studied within the Molecular Electron Density Theory (MEDT) at the $\omega$ B97XD/6-311G(d,p) computational level. Topological analysis of the electron localization function (ELF) of dieniminiums showed that their electronic structures can been seen as the sum of those of butadiene and ethaniminium. The superelectrophilic character of dieniminiums accounts for the high intramolecular global electron density transfer taking place from the diene framework to the iminium one at the transition state structures (TSs) of these IIDA reactions, which are classified as the forward electro density flux. The activation enthalpy associated with the IIDA reaction of the experimental dieniminium, $8.7 \mathrm{kcal} \cdot \mathrm{mol}^{-1}$, was closer to that of the ionic Diels-Alder (I-DA) reaction between butadiene and ethaniminium, $9.3 \mathrm{kcal} \cdot \mathrm{mol}^{-1}$. However, the activation Gibbs free energy of the IIDA reaction was $12.7 \mathrm{kcal} \cdot \mathrm{mol}^{-1}$ lower than that of the intermolecular I-DA reaction. The strong exergonic character of the IIDA reaction, higher than $20.5 \mathrm{kcal} \cdot \mathrm{mol}^{-1}$, makes the reaction irreversible. These IIDA reactions present a total re/exo and si/endo diastereoselectivity, which is controlled by the most favorable chair conformation of the tetramethylene chain. ELF topological analysis of the single bond formation indicated that these IIDA reactions take place through a non-concerted two-stage one-step mechanism. Finally, ELF and atoms-in-molecules (AIM) topological analyses of the TS associated with the inter and intramolecular processes showed the great similarity between them.

Keywords: intramolecular ionic Diels-Alder reactions; molecular electron density theory; dieniminiums; superelectrophiles; global electron density transfer

\section{Introduction}

The Diels-Alder (DA) reaction [1], which is a general class of cycloaddition reactions, is one of the most useful synthetic reactions in Organic Chemistry [2,3]. By varying the nature of the diene and the ethylene, many different types of six-membered carbocyclic structures can be built up. However, not all possibilities take place easily. For instance, the DA reaction between butadiene 1 and ethylene 2 , selected as the prototype of these cycloaddition reactions [4,5], must be forced to take place: after $17 \mathrm{~h}$ at $165^{\circ} \mathrm{C}$ and $900 \mathrm{~atm}$, a yield of $78 \%$ is obtained (see Scheme 1) [6].

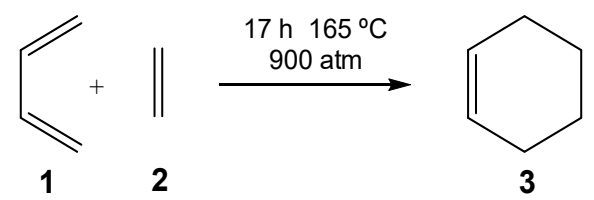

Scheme 1. Diels-Alder (DA) reaction between butadiene $\mathbf{1}$ and ethylene $\mathbf{2}$.

Although this DA reaction is exothermic by $40 \mathrm{kcal} / \mathrm{mol}$, it has a large activation energy of $27.5 \mathrm{kcal} \cdot \mathrm{mol}^{-1}$ [6]. For DA reactions, the negative activation entropy associated with these bimolecular processes also plays an adverse role. In this way, for the DA reaction 
between butadiene 1 and ethylene 2, the activation entropy of the one-step process was estimated to be $-40.6 \mathrm{eu}$ [7]. This unfavorable value, together with the high temperature required by the reaction, increased the activation Gibbs free energy of this DA reaction to $42.6 \mathrm{kcal} \cdot \mathrm{mol}^{-1}$.

There are two different ways to reduce this high activation Gibbs free energy: (i) to decrease the high activation enthalpy associated with the formation of the two new single bonds, and/or (ii) to decrease the high activation entropy associated with these bimolecular processes.

An exhaustive theoretical study on experimental DA reactions, allowed establishing the definitive role of the global electron density transfer [8] (GEDT) taking place at the TSs in the feasibility of DA reactions $[9,10]$. This finding allowed establishing, in 2009, the mechanism of the polar DA (P-DA) reactions [11], in which the favorable nucleophilic/electrophilic interactions taking place at the transition state structures (TSs) are responsible for the feasibility of the reaction.

The very good correlation found between the GEDT and the activation barriers for the DA reactions of $\mathrm{Cp} 4$ with a series of 12 substituted ethylenes, including iminium 5, allowed the classification of the DA reactions in non-polar DA (N-DA) (which do not take place easily experimentally), P-DA reactions, and ionic DA (I-DA) reactions, in which one of the two reagents is an ionic species (see Figure 1) [11]. Although, from the GEDT analysis it is not possible to stablish a clear separation between P-DA and I-DA reactions, these DA reactions have different behaviors.

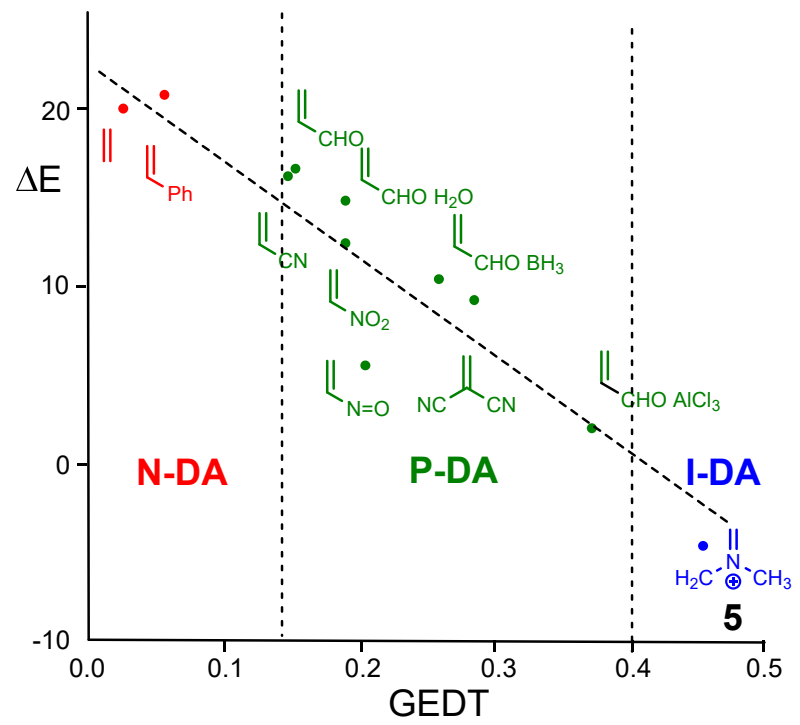

Figure 1. Plot of the activation barriers $\left(\Delta \mathrm{E}\right.$ in $\left.\mathrm{kcal} \cdot \mathrm{mol}^{-1}\right)$ vs. the GEDT, e, $R^{2}=0.89$, for the DA reactions of $C p 4$ with the substituted ethylene series of an increased electrophilic character. The classification of the DA reactions, based on the GEDT, is included.

The I-DA reactions of a series of six iminiums 6 and 7 with Cp 4 have been recently studied within the Molecular Electron Density Theory [12] (MEDT) (see Scheme 2) [13]. The activation energies of these I-DA reactions were found to be between 13 and $20 \mathrm{kcal} \cdot \mathrm{mol}^{-1}$ lower in energy than those associated with the corresponding P-DA reactions of neutral imines as a consequence of the superelectrophilic character of iminiums, $\omega>8.20 \mathrm{eV}$. Unlike P-DA reactions, these I-DA reactions showed a low endo stereoselectivity as a consequence of the cationic character of the TSs; however, they are highly regioselective [13]. In addition, polar solvents have poor effects on the relative energies, and an unappreciable effect in the geometries. 


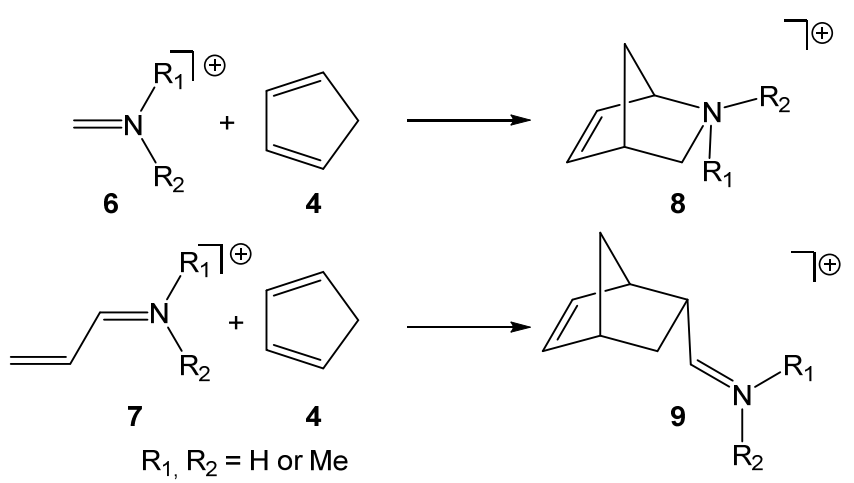

Scheme 2. I-DA reactions of iminiums 6 and 7 with Cp 4.

On the other hand, the high entropy cost associated with bimolecular processes may be overcome through an intramolecular process. Thus, in Table 1, the thermodynamic data for the N-DA reaction between butadiene $\mathbf{1}$ and ethylene 2 given in Scheme 1, and those for the intramolecular Diels-Alder (IMDA) reaction of $(E)$-deca-1,3,9-triene 10 given in Scheme 3 are gathered. The thermodynamic data of all species participating in these N-DA reactions are reported in Table S3 in the Supplementary Materials.

Table 1. B3LYP/6-311G $(\mathrm{d}, \mathrm{p})$ relative enthalpies $\left(\Delta \mathrm{H}\right.$, in $\left.\mathrm{kcal} \cdot \mathrm{mol}^{-1}\right)$, entropies $\left(\Delta \mathrm{S}\right.$, in cal $\left.\cdot \mathrm{mol}^{-1} \cdot \mathrm{K}\right)$ and Gibbs free energies $\left(\Delta \mathrm{G}\right.$, in $\left.\mathrm{kcal} \cdot \mathrm{mol}^{-1}\right)$ computed at $165^{\circ} \mathrm{C}$ and $1 \mathrm{~atm}$ of the stationary points involved in the intermolecular N-DA reaction of butadiene $\mathbf{1}$ and ethylene $\mathbf{2}$, and in the IMDA reaction of (E)-deca-1,3,9-triene $\mathbf{1 0 .}$

\begin{tabular}{cccc}
\hline & $\boldsymbol{\Delta H}$ & $\Delta \mathbf{S}$ & $\Delta \mathbf{G}$ \\
\hline TS-inter & 25.7 & -44.8 & 45.4 \\
$\mathbf{3}$ & -28.5 & -52.4 & -5.5 \\
TS-intra & 29.4 & -19.4 & 38.0 \\
$\mathbf{1 1}$ & -28.8 & -24.2 & -18.2 \\
\hline
\end{tabular}

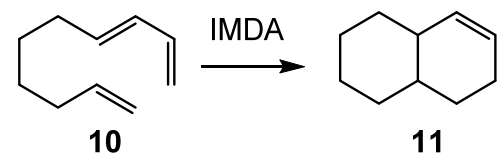

Scheme 3. IMDA reaction of (E)-deca-1,3,9-triene $\mathbf{1 0}$.

The B3LYP/6-311G(d,p) activation enthalpy for the N-DA reaction between butadiene 1 and ethylene $2, \Delta \mathrm{H}^{\#}=25.7 \mathrm{kcal} \cdot \mathrm{mol}^{-1}$, and the activation entropy, $\Delta S^{\#}=-44.8 \mathrm{cal} \cdot \mathrm{mol}^{-1} \cdot \mathrm{K}$, are closer to the experimental values, $27.5 \mathrm{kcal} \cdot \mathrm{mol}^{-1}$ and $-40.6 \mathrm{cal} \cdot \mathrm{mol}^{-1} \cdot \mathrm{K}$, respectively [6,7]. These high values rise the activation Gibbs free energy of this intermolecular $\mathrm{N}-\mathrm{DA}$ reaction computed at $165^{\circ} \mathrm{C}$ to $45.4 \mathrm{kcal} \cdot \mathrm{mol}^{-1}$. Despite the fact that the activation enthalpy associated with the IMDA reaction of $10, \Delta \mathrm{H}^{\#}=29.4 \mathrm{kcal} \cdot \mathrm{mol}^{-1}$, is higher than that of the intermolecular one, the low activation entropy associated with this intramolecular process, $\Delta S^{\#}=-19.4 \mathrm{cal} \cdot \mathrm{mol}^{-1} \cdot \mathrm{K}$, decreases the activation Gibbs free energy to $38.0 \mathrm{kcal} \cdot \mathrm{mol}^{-1}$. This behavior explains the feasibility of non-polar IMDA reactions.

In 1972, R. Sustmann et al. [14] classified the cycloaddition reactions as "the normal and inverse electron-demand (NED and IED)" within the Frontier Molecular Orbital (FMO) theory [15]. Later, in 1973, Houk renamed Sustmann's classification, naming them as (HOMO) HO- and (LUMO) LU-controlled cycloaddition reactions [16], emphasizing the relevance of the molecular orbital (MO) interactions in cycloaddition reactions. However, within MEDT, neither classification based on the FMO theory has any chemical significance. Note that MOs are only mathematical artefact used to construct the wave function.

Thus, for instance, the unfavorable DA reaction between butadiene 1 ethylene 2 given in Scheme 1 is classified as NED or HO-controlled, despite that this non-polar DA reaction 
has a negligible GEDT at the corresponding TS [17]. In addition, many authors have emphasized that these classifications are sometimes confusing, leading to interpretations in contract with the experimental observations [18-21]. On the order hand, in spite of the chemical similarity of the inter and intramolecular cycloaddition reactions, the later cannot be classified within these criteria as the HOMO and LUMO belong to a single molecule.

Recently, Domingo proposed the classification of the polar DA reactions as the forwards and reverse electron density flux (FEDF and REDF) [22]. In FEDF DA reactions, the electron density always fluxes from the diene towards the ethylene. Analysis of the GEDT [8] at the TSs unequivocally allows to classify the DA reactions. Thus, the non-polar DA reaction between butadiene $\mathbf{1}$ and ethylene $\mathbf{2}$ is classified as the null electron density flux (NEDF) [23]. This classification has been applied recently in I-DA reactions [13].

The GEDT measured at the TSs has proved to be a powerful tool to establish the polar character of IMDA reactions [24-26], and consequently, it can be used to classify them depending on the course of the intramolecular electron density flux. Thus, the non-polar IMDA reaction of (E)-deca-1,3,9-triene $\mathbf{1 0}$ given in Scheme 3 is classified as NEDF.

Many theoretical studies devoted to non-polar and polar IMDA reactions can be found in the literature; however, only a small number of them have been devoted to intramolecular ionic Diels-Alder (IIDA) reactions [27,28].

In 1988, Grieco and Parke used the IIDA reaction of dieniminium 12 in the synthesis of (-)-8a-epipumiliotoxin C $\mathbf{1 5}$ (see Scheme 4) [29]. The IIDA reaction of the generated in situ dieniminium 12 provided a mixture of the stereoisomeric quinoliniums $\mathbf{1 3}$ and $\mathbf{1 4}$ in a ratio of 2.2:1. Quinolinium 13 was further converted into $\mathbf{1 5}$ upon a hydrogenation reaction.

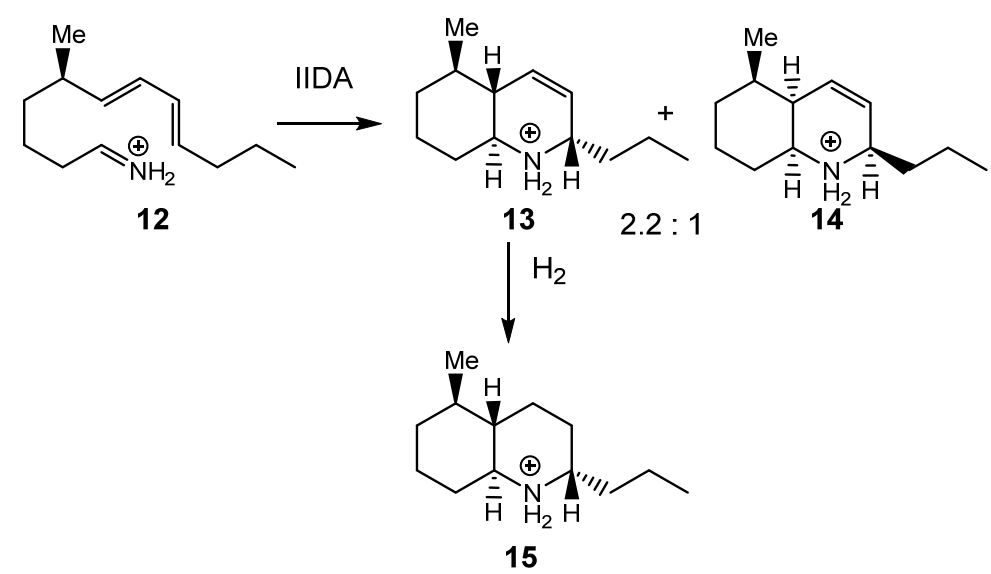

Scheme 4. IIDA reaction of dieniminium 12.

Herein, an MEDT study of the IIDA reaction of dieniminium 12, experimentally studied by Grieco and Parke [29], is performed in order to understand the behaviors of the IIDA reactions (see Scheme 4). To this end, the I-DA reaction of butadiene 1 with iminium 18, and the IIDA reaction of (E)-nona-6,8-dien-1-iminium 16, as a reduced model of dieniminium 12, were also studied (see Scheme 5). The recent classification of cycloaddition reactions based on the direction of the flux of the electron density [22] is for the first time applied in an intramolecular cycloaddition reaction. 
IIDA reactions
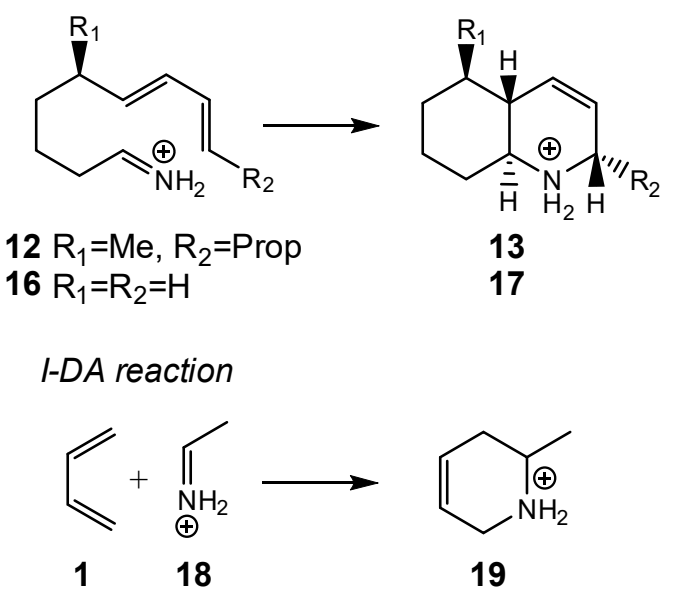

Scheme 5. IIDA reactions of dieniminiums 12 and 16, and I-DA reaction of butadiene 1 with iminium 18 herein studied.

\section{Computational Methods}

DFT calculations were performed using the B3LYP [30,31] functional and hybrid $\omega \mathrm{B} 97 \mathrm{X}-\mathrm{D}$ functional [32], which includes long range exchange (denoted by $\mathrm{X}$ ) correction as well as the semiclassical London-dispersion correction (indicated by suffix-D). The standard 6-311G $(\mathrm{d}, \mathrm{p})$ basis set was used [33], which includes d-type polarization for second row elements and $p$-type polarization functions for hydrogen atoms. The Berny method was used in optimizations [34,35]. Only one imaginary frequency characterized all studied TSs.

The intrinsic reaction coordinate (IRC) paths [36] were carried out to find the unique connection given between the TSs and the minimum stationary points $[37,38]$. Solvent effects of ethanol were considered by full optimization of the gas phase structures at the same computational level using the polarizable continuum model $[39,40]$ (PCM) in the framework of the self-consistent reaction field [41-43] (SCRF).

The GEDT [8] values were estimated by a NPA [44,45], using the equation $\operatorname{DEDT}(\mathrm{f})=\sum_{\mathrm{q} \in \mathrm{f}} \mathrm{q}$, were $\mathrm{q}$ are the atoms of a framework (f) at the TSs. CDFT reactivity indices [46,47], computed at the B3LYP/6-31G(d) level, were calculated through the equations given in reference [47]. All calculations were carried out with the Gaussian 16 suite (Version A.03, Gaussian Inc, Wallingford, CT, USA) of programs [48].

The topology of the electron localization function [49] (ELF) of the B97XD/6-311G(d,p) monodeterminantal wavefunctions was carried out using the TopMod [50] package with a cubical grid of step size of $0.1 \mathrm{Bohr}$. The Bader's quantum theory of atoms-in-molecules [51] (AIM) analyses were conducted using Multiwfn 3.8 software packages [52]. GaussView program [53] (Version 6.0, Semichem Inc., Shawnee, KS, USA) was used to visualize molecular geometries of all the systems as well as the position of the ELF basin attractors. The ELF localization domains at an isovalue of 0.75 a.u. were obtained with the Paraview software (Version 5.6.0 64-bit, Kitware inc, Clifton Park, NY, USA) [54,55].

\section{Results and Discussions}

The present MEDT study was divided into three parts: (i) first, a study of the electronic structure and reactivity at the ground state (GS) of the reagents is performed; (ii) in the second part, the study of the reaction paths associated with I-DA reaction between butadiene 1 and ethaniminium 18, and those associated with the IIDA reactions of dieniminiums 12 and $\mathbf{1 6}$ is carried out; and finally, (iii) in the third part, a topological analysis of the bonding changes along the IIDA reactions of dieniminium 12 is presented. A comparative ELF and AIM topological analysis of the most favorable TSs involved in the inter and intra I-DA reactions is also performed. 


\subsection{Study of the Structure and Reactivity at the GS of the Reagents}

\subsubsection{Analysis of the GS Electronic Structures of the Reagents}

The topological analysis of the ELF [49] allows a quantitative and qualitative characterization of chemical regions of a molecule [56]. Thus, the topology of the ELF of butadiene 1, ethaniminium 18 and dieniminium 16 was first studied in order to characterize their electronic structures. Due to the free $\mathrm{C}-\mathrm{C}$ single bond rotation of the tetramethylene chain of dieniminium 16, this species can be found as a series of conformers, also named rotamers.

Only two conformations were selected in the present study: (i) the extended conformation, denoted as 16e, in which all methylenes $\mathrm{CH}_{2}$ of the tetramethylene chain are positioned in alternate conformations; and (ii) the fold conformation, denoted as $\mathbf{1 6 f}$, which opens the reaction path for the IIDA reaction. The ELF basin attractor positions of $\mathbf{1}, \mathbf{1 8}$, and 16e are shown in Figure 2. The ELF basin attractor positions of dieniminium 12e are shown in Figure S3 in the Supplementary Materials.

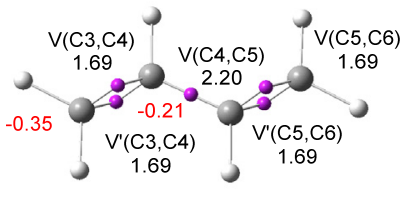

1

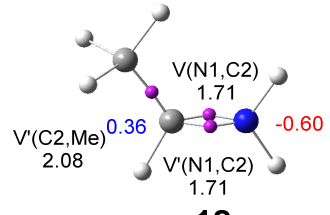

18

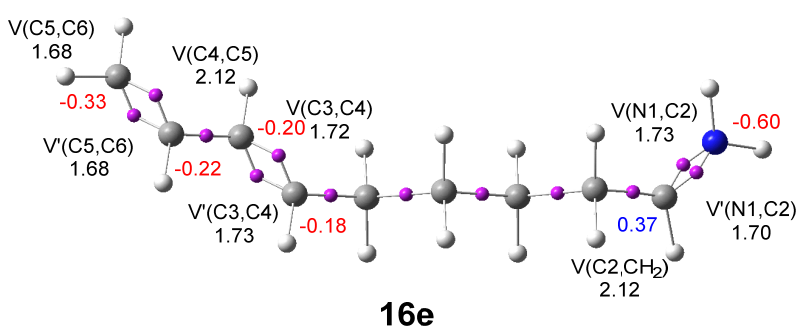

Figure 2. ELF basin attractor positions together with the most relevant valence basin populations, and natural atomic charges, in average number of electrons e, blue color is used for positive charges and red color for negative ones, of butadiene 1, ethaniminium 18 and dieniminium 16e.

The ELF analysis of butadiene 1 shows the presence of two pairs of disynaptic basins, $\mathrm{V}(\mathrm{C} 3[5], \mathrm{C} 4[6])$ and $\mathrm{V}^{\prime}(\mathrm{C} 3[5], \mathrm{C} 4[6])$, integrating a total population of 3.38 e each one, associated with two depopulated $\mathrm{C} 3[5]-\mathrm{C} 4[6]$ double bonds, and one $\mathrm{V}(\mathrm{C} 4, \mathrm{C} 5)$ disynaptic basin, integrating $2.20 \mathrm{e}$, associated with a populated C4-C5 single bond. On the other hand, the ELF analysis of ethaniminium 18 shows the presence of two disynaptic basins, $\mathrm{V}(\mathrm{N} 1, \mathrm{C} 2)$ and $\mathrm{V}^{\prime}(\mathrm{N} 1, \mathrm{C} 2)$, integrating a total of $3.42 \mathrm{e}$, associated with a depopulated N1-C2 double bond.

The ELF analysis of dieniminium 16e shows the presence of two disynaptic basin, $\mathrm{V}(\mathrm{N} 1, \mathrm{C} 2)$ and $\mathrm{V}^{\prime}(\mathrm{N} 1, \mathrm{C} 2)$, integrating a total of 3.43 e associated with the $\mathrm{N} 1-\mathrm{C} 2$ double bond of the iminium framework, and two pairs of disynaptic basins, $\mathrm{V}(\mathrm{C} 3[5], \mathrm{C} 4[6])$ and $\mathrm{V}^{\prime}(\mathrm{C} 3[5]$, $\mathrm{C} 4[6])$, integrating a total population of ca 3.4 e each one, and one $\mathrm{V}(\mathrm{C} 4, \mathrm{C} 5)$ disynaptic basin, integrating $2.12 \mathrm{e}$, associated with the butadiene $\mathrm{C} 3-\mathrm{C} 4-\mathrm{C} 5-\mathrm{C} 6$ framework.

The tetramethylene chain joining the butadiene and iminium frameworks is characterized by the presence of three $\mathrm{V}(\mathrm{Ci}, \mathrm{Cj})$ disynaptic basins, integrating between $1.70 \mathrm{e}$ and $1.86 \mathrm{e}$. ELF of dieniminium 16e indicates that the electronic structure of this species can be seen as the addition of the electronic structure of butadiene $\mathbf{1}$, plus that of ethaniminium 18, plus that of the tetramethylene chain. ELF of experimental dieniminium 12e is identical to that of 16e (see Figure S3 in the Supplementary material).

The natural atomic charges [44,45] are given in Figure 2. At ethaniminium 18, while the $\mathrm{C} 2$ carbon is positively charged by $0.36 \mathrm{e}$, the $\mathrm{N} 1$ nitrogen is negatively charged by -0.60 e [13]. At dieniminium 16e, the N1 nitrogen and C3 carbon present the same charge distribution than that at ethaniminium 18. The only difference is that the $\mathrm{C} 3$ carbon of dieniminium 16e presents a lower negative charge, -0.18 , as a consequence of the 
substitution of a hydrogen at ethaniminium 18 by a methylene at 16e. Interestingly, while the Lewis structures of iminiums localize the positive charge at the nitrogen atom, it is negatively charged [13].

\subsubsection{Analysis of the CDFT Reactivity Indices at the GS of the Reagents}

Many studies devoted to cycloaddition reactions have proof that the analysis of the global and local reactivity indices defined within the CDFT $[46,47]$ is a powerful tool to understand polar reactions $[57,58]$. The CDFT indices were obtained at the B3LYP/6$31 \mathrm{G}(\mathrm{d})$ computational level due to being used to define the scales of electrophilicity and nucleophilicity [47]. The global reactivity indices of the reagents participating in the I-DA and IIDA reactions herein studied are collected in Table 2.

Table 2. B3LYP/6-31G(d) global reactivity indices; the electronic chemical potential, $\mu$; chemical hardness, $\eta$; electrophilicity, $\omega$; and nucleophilicity, $N$; in $\mathrm{eV}$, of the reagents participating in the I-DA and IIDA reactions herein studied.

\begin{tabular}{ccccc}
\hline & $\boldsymbol{\mu}$ & $\boldsymbol{\eta}$ & $\boldsymbol{\omega}$ & $\boldsymbol{N}$ \\
\hline 12e & -7.39 & 1.02 & 26.72 & 1.22 \\
16e & -7.65 & 1.16 & 25.19 & 0.89 \\
16f & -8.23 & 3.76 & 9.01 & -0.99 \\
ethaniminium 18 & -11.82 & 8.27 & 8.46 & -6.83 \\
12f & -7.85 & 3.68 & 8.36 & -0.57 \\
butadiene 1 & -3.42 & 5.62 & 1.04 & 2.89 \\
pentadiene 20 & -3.17 & 5.51 & 0.91 & 3.20 \\
dienimine 21 & -3.31 & 5.47 & 1.00 & 3.08 \\
\hline
\end{tabular}

The electronic chemical potential $[46,59] \mu$ of butadiene $1, \mu=-3.42 \mathrm{eV}$, is higher than that of ethaniminium 18, $\mu=-11.82 \mathrm{eV}$. Consequently, it is expected that along the corresponding I-DA reaction, an amount of the electron density will be transferred from butadiene 1 to ethaniminium 18, with the reaction being classified as forward electron density flux (FEDF) [22].

The electrophilicity index [60] of the iminiums ranges from 8.36 (12f) to 26.72 (12e) $\mathrm{eV}$, being classified as strong electrophiles within the electrophilicity scale [47]. The electrophilicity $\omega$ index of iminium $\mathbf{1 2 f}, 8.36 \mathrm{eV}$, is closer to that of ethaniminium $\mathbf{1 8}, 8.46 \mathrm{eV}$, being both classified as superelectrophiles [61]. As expected, the nucleophilicity $N$ index [62] of these species range from $-6.83(\mathbf{1 8})$ to $1.12(\mathbf{1 2 e}) \mathrm{eV}$, being classified as marginal nucleophiles within the nucleophilicity scale [47].

Interestingly, the extended conformations of dieniminiums 12e and 16e present unusual electrophilicity $\omega$ values, 26.72 (12e) and 25.19 (16e) eV, respectively, when they are compared with those of the fold conformations 12f and 16f. Analysis of the reactivity indices of these species indicates that they present a very low hardness, less than $1.20 \mathrm{eV}$ (see Table 2). As the hardness $\eta$ is found in the denominator of the equation defining the electrophilicity $\omega$ index (see Equation (1)) [60], a decrease of the hardness $\eta$ provokes a noticeable increase of the electrophilicity $\omega$ index.

On the other hand, the hardness $\eta$ is approaches the HOMO-LUMO gap (see Equation (2)) [46]. The presence of the iminium and the diene frameworks in these species decreases and notably increases the LUMO and HOMO energies, respectively, thus, causing an unexpected very low value for the hardness $\eta$, and, consequently, a very high value for the electrophilicity $\omega$ index.

$$
\begin{gathered}
\omega=\frac{\mu^{2}}{2 \eta} \\
\eta \approx\left(\mathrm{E}_{\mathrm{LUMO}}-\mathrm{E}_{\mathrm{HOMO}}\right)
\end{gathered}
$$

A good correlation between the electrophilicity $\omega$ indices and the LUMO energies, $\varepsilon_{\text {LUMO }}$, for the ten substituted ethylenes given in Figure 1 is established in Section 1 in the 
Supplementary Materials. This correlation allows establishing the polynomic Equation (3), which permits us to estimate the electrophilicity $\omega$ through LUMO energies, $\varepsilon_{\text {LUMO: }}$

$$
\omega_{\mathrm{LUMO}}=0.059 * \varepsilon_{\mathrm{LUMO}}^{2}-0.5803 * \varepsilon_{\mathrm{LUMO}}+0.8589
$$

By using the proposed Equation (3), the electrophilicity $\omega_{\text {LUMO }}$ index for the extended conformations of dieniminiums $\mathbf{1 2}$ and $\mathbf{1 6}$ are estimated as $7.64 \mathrm{eV}$ for $\mathbf{1 2 e}$, and $7.91 \mathrm{eV}$ for 16e (see Table S2), which are in reasonable agreement with the values computed for the fold conformations of dieniminiums 12 and 16: $8.36 \mathrm{eV}$ or $\mathbf{1 2 f}$ and $9.91 \mathrm{eV}$ for $\mathbf{1 6 f}$.

Butadiene 1 has an electrophilicity $\omega$ and nucleophilicity $N$ indices of 1.04 and $2.89 \mathrm{eV}$, being classified as a moderate electrophile and in the borderline of moderate nucleophile. The inclusion of an electron-releasing methyl group on butadiene increases the nucleophilicity $\mathrm{N}$ index of pentadiene 20 to $3.20 \mathrm{eV}$, being classified as a strong nucleophile. This value is closer to that of dienimine $21, N=3.08 \mathrm{eV}$, the deprotonated iminium 16 . Consequently, the I-DA reaction between ethaniminium 18 and pentadiene 20 will also be characterized by a high GEDT.

DA reactions involving asymmetric electrophilic compounds take place via asynchronous TSs $[10,13]$. The most favorable two-center interactions between the two interacting species in polar and ionic processes take place between the most electrophilic center of the electrophile and the most nucleophilic center of the nucleophile [63]. In this context, the electrophilic $P_{k}^{+}$and nucleophilic $P_{k}^{-}$Parr functions [64] derived from the excess of spin electron density reached via the GEDT [8] process from the nucleophile toward the electrophile have shown to be one of the most accurate and insightful tools for the study of the local reactivity in polar and ionic processes.

On the other hand, analysis of the electrophilic $P_{k}^{+}$and nucleophilic $P_{k}^{-}$Parr functions in intramolecular cycloaddition reactions allows characterizing the electrophilic and nucleophilic centers of the molecules [65]. Consequently, the electrophilic $P_{k}^{+}$and nucleophilic $P_{k}^{-}$Parr functions of the model dieniminium 16e were calculated and analyzed. The corresponding values are given in Figure 3.
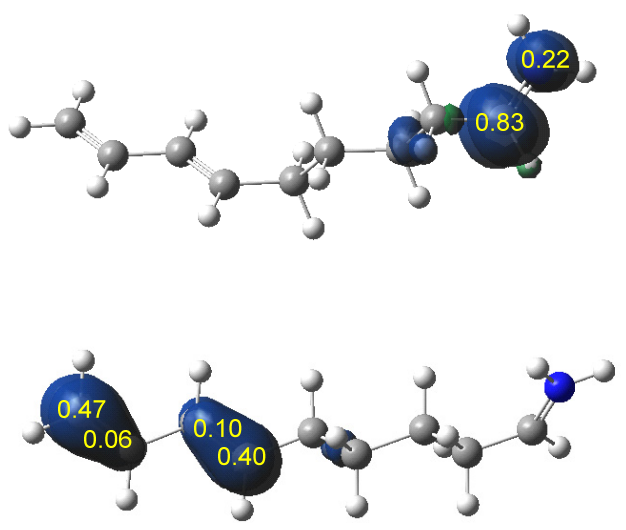

Figure 3. 3D representations of the Mulliken atomic spin densities of the neutral radical and radical cation of the reduced model dieniminium 16e and the electrophilic $P_{k}^{+}$and nucleophilic $P_{k}^{-}$Parr functions.

Analysis of the electrophilic $P_{k}^{+}$Parr functions of dieniminium 16e shows that they are manly concentrated on the $\mathrm{N}=\mathrm{C}$ iminium framework. Thus, the iminium $\mathrm{C} 2$ carbon, with a $P_{k}^{+}=0.83$, concentrates more than the $80 \%$ of the electrophilicity of this species. On the other hand, the nucleophilic $P_{k}^{-}$Parr functions of dieniminium 16e shows that they are mainly concentrated on the $\mathrm{C} 3, P_{k}^{-}=0.40$ and $\mathrm{C} 6, P_{k}^{-}=0.47$, carbons of the butadiene framework. Although the most favorable two-center interactions along the IIDA reaction are those between the $\mathrm{C} 2$ and $\mathrm{C} 6$ centers, this will provoke a strong geometrical distortion. Consequently, along the intramolecular process, the best two-center interaction takes place between the $\mathrm{C} 2$ and $\mathrm{C} 3$ centers. 
While the electrophilicity $\omega$ index classifies dieniminium 16e as a supernucleophile, which will experience a high intramolecular GEDT at the TS of the corresponding IIDA reaction (see later), the analysis of the electrophilic $P_{k}^{+}$and nucleophilic $P_{k}^{-}$Parr functions unequivocally characterizes the electrophilic and nucleophiles frameworks of dieniminium 16e. This behavior allows predicting that the corresponding IIDA reaction will be classified as FEDF [22].

\subsection{Study of the I-DA Reaction between Butadiene 1 and Ethaniminium 18, and the IIDA Reactions of Dieniminiums 12 and 16}

3.2.1. Study of the I-DA Reaction between Butadiene 1 and Ethaniminium 18

In order to understand the IIDA reaction of dieniminium 12 (see Scheme 6), the I-DA reaction between butadiene 1 and ethaniminium 18 was first studied. This I-DA reaction can take place via two stereoisomeric reaction paths, the endo and the exo. Along the endo approach mode, the methyl group of ethaniminium 18 is positioned above the diene system of butadiene 1 . Analysis of the stationary points found along the two reaction paths indicates that this I-DA reaction takes place through a one-step mechanism. Consequently, two TSs, TS1-n and TS1-x, and the corresponding cycloadducts 22 and $\mathbf{2 3}$ were located and characterized. The relative energies in ethanol are given in Table 3, while the total energies are given in Table S4 in the Supplementary Materials.

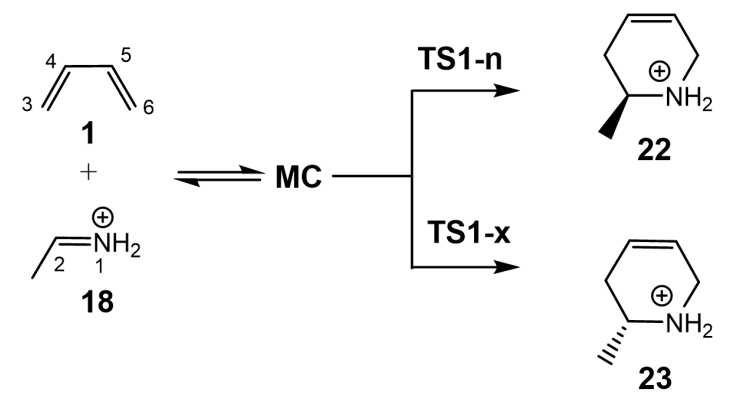

Scheme 6. I-DA reaction between butadiene 7 and ethaniminium 8 .

Table 3. $\omega \mathrm{B} 97 \mathrm{XD} / 6-311 \mathrm{G}(\mathrm{d}, \mathrm{p})$ relative electronic energies $\left(\Delta \mathrm{E}\right.$, in $\left.\mathrm{kcal} \cdot \mathrm{mol}^{-1}\right)$, enthalpies $(\Delta \mathrm{H}$, in $\left.\mathrm{kcal} \cdot \mathrm{mol}^{-1}\right)$, entropies $(\Delta \mathrm{S}$, in cal $\cdot \mathrm{mol} . \mathrm{K})$, and Gibbs free energies $\left(\Delta \mathrm{G}\right.$, in $\left.\mathrm{kcal} \cdot \mathrm{mol}^{-1}\right)$ computed at $75^{\circ} \mathrm{C}$ and $1 \mathrm{~atm}$ in ethanol, of the stationary points involved in the I-DA reactions of butadiene 1 with ethaniminium 18.

\begin{tabular}{ccccc}
\hline & $\boldsymbol{\Delta} \mathbf{E}$ & $\boldsymbol{\Delta H}$ & $\boldsymbol{\Delta} \mathbf{S}$ & $\boldsymbol{\Delta} \mathbf{G}$ \\
\hline $\mathbf{M C}$ & -4.5 & -3.1 & -26.0 & 6.0 \\
TS1-n & 8.1 & 9.3 & -46.1 & 25.3 \\
TS1-x & 9.0 & 10.1 & -44.9 & 25.8 \\
$\mathbf{2 2}$ & -41.3 & -36.3 & -51.6 & -18.3 \\
$\mathbf{2 3}$ & -35.9 & -31.0 & -49.9 & -13.6 \\
\hline
\end{tabular}

At the beginning of the reaction, a series of molecular complexes (MCs) can be found. They are characterized by the presence of weak electronic interactions between butadiene 1 and iminium 18. Only the most stable MC was selected as the energy reference. MC is found $4.5 \mathrm{kcal} \cdot \mathrm{mol}^{-1}$ below the separated reagents. The relative enthalpies of the TSs with respect the reagents are 8.1 (TS1-n) and 9.0 (TS1-x) $\mathrm{kcal} \cdot \mathrm{mol}^{-1}$; the reaction being exothermic by more than $35.9(23) \mathrm{kcal} \cdot \mathrm{mol}^{-1}$.

Some appealing conclusions can be drawn from the energy results given in Table 3: (i) the formation of $\mathrm{MC}$ is an exothermic process. However, if the thermal correction and the entropies are considered, they become endergonic $6.0 \mathrm{kcal} \cdot \mathrm{mol}^{-1}$; (ii) in ethanol, this I-DA reaction presents an activation energy of $8.1 \mathrm{kcal} \cdot \mathrm{mol}^{-1}$. Note that, in the gas phase, the TSs are found below the separated reagents [13]; (iii) this I-DA reaction presents a low 
endo stereoselectivity as TS1-x is found only $0.9 \mathrm{kcal} \cdot \mathrm{mol}^{-1}$ above TS1-n; and (iv) this I-DA reaction is highly exothermic; higher than $36 \mathrm{kcal} \cdot \mathrm{mol}^{-1}$.

The thermodynamic data of the I-DA reaction between butadiene 1 and ethaniminium 18 were then analyzed. The relative enthalpies, entropies, and Gibbs free energies, computed at $75{ }^{\circ} \mathrm{C}$ in ethanol, are given in Table 3. The inclusion of the thermal corrections to the electronic energies in ethanol increases the relative enthalpies between 1.1 and $5.1 \mathrm{kcal} \cdot \mathrm{mol}^{-1}$. The lower incidence takes place in the relative enthalpies of TSs, which increase by only $1.1 \mathrm{kcal} \cdot \mathrm{mol}^{-1}$ with respect to the electronic energies in ethanol. The inclusion of the thermal corrections and entropies to enthalpies increases the relative Gibbs free energies between 8 and $18 \mathrm{kcal} \cdot \mathrm{mol}^{-1}$ as a consequence of the high activation entropy associated with this bimolecular process, between -26 and $-46 \mathrm{cal} \cdot \mathrm{mol}^{-1} \cdot \mathrm{K}^{-1}$.

Thus, the activation Gibbs free energy associated with this I-DA reaction in ethanol rises to $25.3 \mathrm{kcal} \cdot \mathrm{mol}^{-1}$, while the formation of 22 becomes exergonic by $-18.3 \mathrm{kcal} \cdot \mathrm{mol}^{-1}$. The activation Gibbs free energy associated with this I-DA reaction is $20.1 \mathrm{kcal} \cdot \mathrm{mol}^{-1}$ lower than that associated with the N-DA reaction between butadiene 1 and ethylene 2 (see Table 1) as a consequence of the high GEDT taking place at the I-DA reaction (see later) [13]. It is important to remark that, while the formation of $\mathbf{M C}$ is exothermic by $3.1 \mathrm{kcal} \cdot \mathrm{mol}^{-1}$, it is endergonic by $6.0 \mathrm{kcal} \cdot \mathrm{mol}^{-1}$ as a consequence of the high negative entropy associated with its formation: $-26.0 \mathrm{cal} \cdot \mathrm{mol}^{-1} \cdot \mathrm{K}^{-1}$ (see Table 3 ).

The geometries of TS1-n and TS1-x involved in the I-DA reaction of butadiene 1 with ethaniminium 18 are given in Figure 4. The distances between the $\mathrm{C} 2-\mathrm{C} 3$ and N1-C6 interacting centers at the TSs are: 1.894 and $2.761 \AA$ at TS1-n and 1.905 and $2.793 \AA$ at TS1-x. These geometrical parameters indicate that these TSs are associated with high asynchronous single bond formation processes. Considering that the $\mathrm{C}-\mathrm{C}$ single bond formation takes place in the range 2.0-1.9 $[$ [8], the C2-C3 distances indicate that at these TSs the formation of the corresponding $\mathrm{C} 2-\mathrm{C} 3$ single bond has begun.

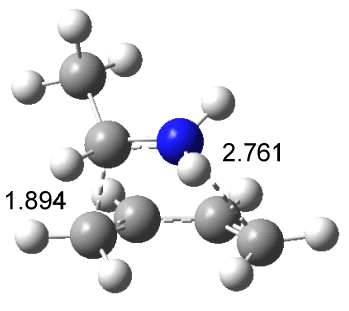

TS1-n

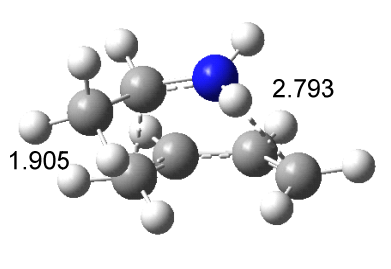

TS1-x

Figure 4. $\omega B$ 97XD/6-311G $(\mathrm{d}, \mathrm{p})$ geometries of TS1-n and TS1-x. The C-C and C-N distances are given in Angstroms.

The ionic nature of this I-DA reaction was evaluated by computing the GEDT values at the TSs. The computed GEDT values at the corresponding TSs, which fluxes from the nucleophilic butadiene $\mathbf{1}$ to the superelectrophilic iminium $\mathbf{1 8}$ are $0.46 \mathrm{e}$ at TS1-n and TS1-x. These very high values indicate the high GEDT taking place at these I-DA reactions, being classified as FEDF [22].

\subsubsection{Study of the IIDA Reactions of Dieniminiums $\mathbf{1 2}$ and $\mathbf{1 6}$}

The IIDA reactions of dieniminiums $\mathbf{1 2}$ and $\mathbf{1 6}$ can take place through four competitive reaction paths (see Scheme 7). They are related to the endo and exo approach modes of the iminium framework with respect to the diene system, named as $n$ and $x$, and through the pro-re and pro-si faces of the C3 carbon of the butadiene system, named as $r$ and $s$. 


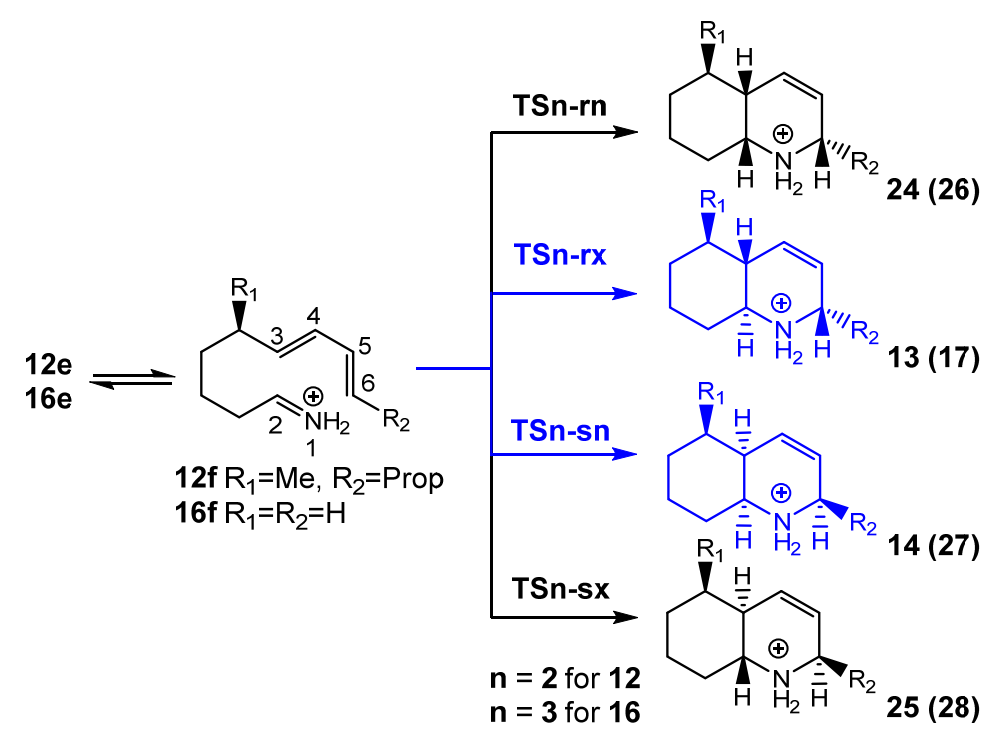

Scheme 7. Competitive reaction paths associated with the IIDA reactions of dieniminiums 12 and 16.

Analysis of the stationary points found along the four reaction paths indicates that these IIDA reactions take place through a one-step mechanism. Consequently, four TSs, TSn-rn, TSn-rx, TSn-sn, and TSn-sx, were $n=2$ for 12 and $n=3$ for 16, and the corresponding quinoliniums were located and characterized for each IIDA reaction. The relative energies in ethanol are given in Table 4, while the total energies are given in Table S5 Supplementary Materials.

Table 4. $\omega B$ 97XD/6-311G $(\mathrm{d}, \mathrm{p})$ relative energies $\left(\Delta \mathrm{E}\right.$, in $\mathrm{kcal} \cdot \mathrm{mol}^{-1}$, with respect to the most stable conformation) in ethanol of the stationary points involved in the IIDA reactions of dieniminiums 12 and $\mathbf{1 6 .}$

\begin{tabular}{cccc}
\hline & $\boldsymbol{\Delta E}$ & & $\Delta \mathrm{E}$ \\
\hline $\mathbf{1 2 e}$ & 0.3 & $\mathbf{1 6 e}$ & 0.0 \\
$\mathbf{1 2 f}$ & 0.0 & $\mathbf{1 6 f}$ & 1.0 \\
TS2-rn & 12.9 & TS3-rn & 17.7 \\
TS2-rx & 8.8 & TS3-rx & 13.6 \\
TS2-sn & 8.9 & TS3-sn & 13.6 \\
TS2-sx & 13.9 & TS3-sx & 18.1 \\
$\mathbf{2 4}$ & -25.0 & $\mathbf{2 6}$ & -27.1 \\
$\mathbf{1 3}$ & -31.1 & $\mathbf{1 7}$ & -30.6 \\
$\mathbf{1 4}$ & -30.3 & $\mathbf{2 7}$ & -30.1 \\
$\mathbf{2 5}$ & -25.2 & $\mathbf{2 8}$ & -24.5 \\
\hline
\end{tabular}

Dieniminiums 12 and 16 can exist in several conformations resulting from the free rotation of the $\mathrm{C}-\mathrm{C}$ single bonds of the tetramethylene chain. For these dieniminiums, two conformations were considered; the fold $12 \mathrm{f}$ and $\mathbf{1 6 f}$, and the extended $12 \mathrm{e}$ and $16 \mathrm{e}$ conformations. The activation energies in ethanol associated with these IIDA reactions ranges

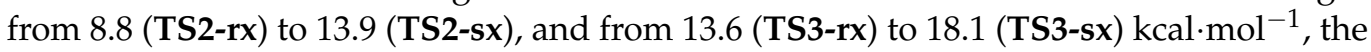
reactions being strongly exothermic by more than $25 \mathrm{kcal} \cdot \mathrm{mol}^{-1}$.

Some appealing conclusions can be obtained from the relative energies given in Table 4: (i) for the experimental dieniminium 12, the fold conformation $\mathbf{1 2 f}$ is found $0.3 \mathrm{kcal} \cdot \mathrm{mol}^{-1}$ below the extended conformation $\mathbf{1 2} \mathbf{e}$; for the dieniminium model $\mathbf{1 6}$, the extended conformation $16 \mathrm{e}$ is found $1.0 \mathrm{kcal} \cdot \mathrm{mol}^{-1}$ below the fold conformation $16 \mathrm{f}$; (ii) the activation energy associated with the IIDA reaction of experimental dieniminium 12, 8.8 (TS2-rx) $\mathrm{kcal} \cdot \mathrm{mol}^{-1}$, is $4.8 \mathrm{kcal} \cdot \mathrm{mol}^{-1}$ lower in energy than that associated with the IIDA

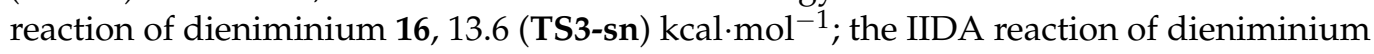
12 presents a closer activation energy than that of the intermolecular process (see Table 3); 
(iii) these IIDA reactions present a high exo selectivity along the re approach mode and a high endo selectivity along the si approach mode; (iv) the formation of quinolinium 13 via TS2-rx is only $0.1 \mathrm{kcal} \cdot \mathrm{mol}^{-1}$ more favorable than the formation of quinolinium 14 via TS2sn, while the formation of quinoliniums $\mathbf{2 4}$ and $\mathbf{2 5}$ is kinetically unfavored in agreement with the experimental outcomes [29]; and finally, (v) the formation of quinoliniums $\mathbf{1 3}$ and 14 is exothermic by more than $30 \mathrm{kcal} \cdot \mathrm{mol}^{-1}$.

The thermodynamic data of the IIDA reaction of experimental dieniminium 12 were analyzed. Relative enthalpies, entropies and Gibbs free energies, computed at $75{ }^{\circ} \mathrm{C}$ in ethanol, are given in Table 5. The inclusion of the thermal corrections to the electronic energies in ethanol modifies the relative enthalpies between -1.3 and $2.8 \mathrm{kcal} \cdot \mathrm{mol}^{-1}$. The lower incidence is found in the relative enthalpies of the TSs, which decrease by less than $1.3 \mathrm{kcal} \cdot \mathrm{mol}^{-1}$ with respect to the electronic energies in ethanol.

Table 5. $\omega \mathrm{B} 97 \mathrm{XD} / 6-311 \mathrm{G}(\mathrm{d}, \mathrm{p})$ relative enthalpies $\left(\Delta \mathrm{H}\right.$, in $\left.\mathrm{kcal} \cdot \mathrm{mol}^{-1}\right)$, entropies $\left(\Delta \mathrm{S}\right.$, in cal $\left.\cdot \mathrm{mol}^{-1} \cdot \mathrm{K}\right)$, and Gibbs free energies $\left(\Delta \mathrm{G}\right.$, in $\left.\mathrm{kcal} \cdot \mathrm{mol}^{-1}\right)$ with respect to the most stable conformation $\mathbf{1 2 f}$, computed at $75{ }^{\circ} \mathrm{C}$ and $1 \mathrm{~atm}$ in ethanol, of the stationary points involved the IIDA reactions of dieniminium 12.

\begin{tabular}{cccc}
\hline & $\boldsymbol{\Delta H}$ & $\boldsymbol{\Delta S}$ & $\boldsymbol{\Delta} \mathbf{G}$ \\
\hline $\mathbf{1 2 e}$ & 0.0 & 0.0 & 0.0 \\
$\mathbf{1 2 f}$ & -1.1 & -11.2 & 2.8 \\
TS2-rn & 12.0 & -16.2 & 17.6 \\
TS2-rx & 7.6 & -14.3 & 12.6 \\
TS2-sn & 7.9 & -16.6 & 13.7 \\
TS2-sx & 13.0 & -14.5 & 18.1 \\
$\mathbf{2 4}$ & -22.2 & -23.8 & -14.0 \\
$\mathbf{1 3}$ & -28.5 & -23.1 & -20.5 \\
$\mathbf{1 4}$ & -27.8 & -20.9 & -20.6 \\
$\mathbf{2 5}$ & -22.3 & -22.8 & -14.4 \\
\hline
\end{tabular}

The inclusion of the thermal corrections and entropies to enthalpies increases the relative Gibbs free energies between 3.9 and $8.3 \mathrm{kcal} \cdot \mathrm{mol}^{-1}$ as a consequence of the negative activation entropy associated with this intramolecular process, between -11.2 and $-23.8 \mathrm{cal} \cdot \mathrm{mol}^{-1} \cdot \mathrm{K}^{-1}$. Thus, the activation Gibbs free energy associated with this IIDA reaction in ethanol rises to $12.6 \mathrm{kcal} \cdot \mathrm{mol}^{-1}$, while the formation of $\mathbf{1 3}$ becomes exergonic by $-20.5 \mathrm{kcal} \cdot \mathrm{mol}^{-1}$.

The activation enthalpy associated with the IIDA reaction via TS2-rx is $0.7 \mathrm{kcal} \cdot \mathrm{mol}^{-1}$ higher than that associated with the intermolecular I-DA reaction via TS1-n, while the activation Gibbs free energy associated with the IIDA reaction is $12.7 \mathrm{kcal} \cdot \mathrm{mol}^{-1}$ lower than that associated with the intermolecular I-DA reaction (see Table 3). The lower entropy loss taking place along the IIDA reaction of the experimental dieniminium $12,-14.3 \mathrm{cal} \cdot \mathrm{mol}^{-1} \cdot \mathrm{K}$, compared with that associated with intermolecular process, $-46.1 \mathrm{cal} \cdot \mathrm{mol}^{-1} \cdot \mathrm{K}$, is responsible for the high acceleration found in the intramolecular process.

The geometries of the TSs involved in the IIDA reaction of dieniminium $\mathbf{1 2 f}$ are given in Figure 5, while those involved in the IIDA reaction of dieniminium 16f are given in Figure S4 in the Supplementary Materials. The distances between the C2-C3 and N1-C6 interacting centers at the TSs involved in the IIDA reactions of dieniminium $12 \mathrm{f}$ are found in a narrow range: between 1.93 and $1.98 \AA(\mathrm{C} 2-\mathrm{C} 3)$ and 2.81 and $3.05 \AA(\mathrm{N} 1-\mathrm{C} 6)$. 


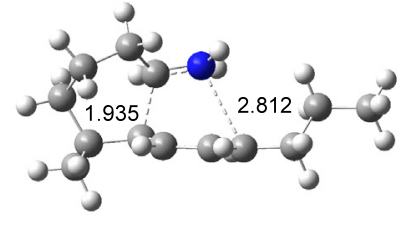

TS2-rn

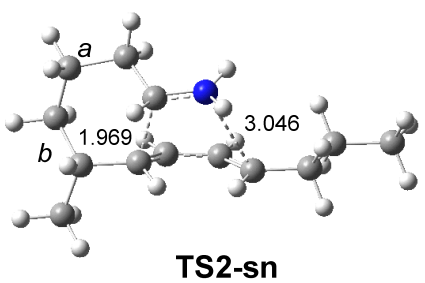

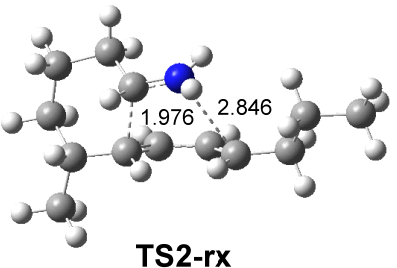

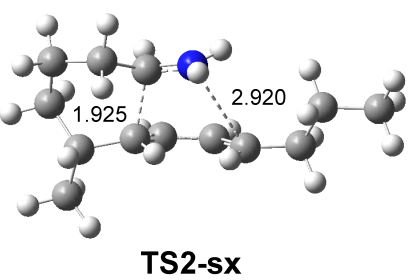

Figure 5. $\omega \mathrm{B} 97 \mathrm{XD} / 6-311 \mathrm{G}(\mathrm{d}, \mathrm{p})$ geometries of the TSs involved in the IIDA reaction of experimental dieniminium 12. The $\mathrm{C}-\mathrm{C}$ and $\mathrm{C}-\mathrm{N}$ distances are given in Angstroms.

Some appealing conclusions can be obtained for these geometrical parameters: (i) these distances indicate that these TSs are associated with high asynchronous single bond formation processes; (ii) the more favorable TS2-rx and TS2-sn are slightly earlier; (iii) these distances associated with the IIDA reaction are very closer to those found at the I-DA reaction of butadiene 1 with ethaniminium 18 (see Figure 4); (iv) while, at the more favorable TS2-rx and TS2-sn, the tetramethylene chain adopts a six-membered chair conformation, at the more unfavorable TS2-rn and TS2-sx, it adopts a six-membered boat conformation. Consequently, the stereochemistry of these IIDA reactions is determined by the arrangement of the tetramethylene chain, thus justifying the total re/exo and si/endo facial diastereoselectivity found in these IIDA reactions with respect to low endo stereoselectivity found in I-DA reactions [13]; (v) while the most favorable re/exo reaction path is associated with the formation of the trans quinolinium 13, the si/endo reaction path is associated with the formation of the cis quinolinium 14. These behaviors account for the fact that formation of trans quinolinium 13 is $0.5 \mathrm{kcal} \cdot \mathrm{mol}^{-1}$ more exergonic than formation of cis quinolinium 14; and finally (vi) a comparison between the geometries of the TSs of the IIDA reactions of dieniminiums $\mathbf{1 2}$ and $\mathbf{1 6}$ indicates that the TSs associated with the reaction model are only slightly more advanced as less asynchronous than those associated with the experimental one (see Figure S4 in the Supplementary Materials).

The ionic nature of the IIDA reaction of dieniminium 12 was evaluated by computing the GEDT at the TSs. To this end, the natural atomic charges at the four TSs were shared in two groups through the two central $\mathrm{Ca}$ and $\mathrm{Cb}$ carbons of the tetramethylene chain (see Figure 5). The computed intramolecular GEDT values at the corresponding TSs, which fluxes from the nucleophilic butadiene framework to the superelectrophilic iminium one, are: 0.45 e (TS2-rn), 0.44 (TS2-rx), 0.43 (TS2-sn) and 0.49 (TS2-sx).

These very high values, which are very similar to those obtained at the I-DA reaction of butadiene 1 with ethaniminium 18, point out the high intramolecular GEDT taking place at these IIDA reactions. The intramolecular electron density flux of this IIDA reaction, which similar to that in the I-DA reaction between butadiene $\mathbf{1}$ to the superelectrophilic iminium 18 takes place from the butadiene framework to the iminium one, allows to classify this IIDA reaction as FEDT [22], a behavior that can be predicted by analysis of the electrophilic $P_{k}^{+}$and nucleophilic $P_{k}^{-}$Parr functions.

\subsection{Topological Analysis of the Bonding Changes along the IIDA Reactions}

3.3.1. ELF Analysis of the N1-C6 and C2-C3 Single Bond Formation along the IIDA Reaction of Dieniminium 16

In order to characterize the formation of the two new $\mathrm{N} 1-\mathrm{C} 6$ and $\mathrm{C} 2-\mathrm{C} 3$ single bonds along the most favorable re/exo reaction path associated with the IIDA reaction of dieniminium 16, and thus, to establish the molecular mechanism of these IIDA reactions, 
a topological analysis of the ELF of the three more relevant structures involved in the formation of the two new single bonds was performed.

These three structures S5-S7 were selected from a Bonding Evolution Theory [66] (BET) study of the IIDA reaction of dieniminium 16. S5-S7 correspond to the first structure characterizing Phases V-VII (see Table S7 in the Supplementary Material). The attractor positions of the ELF basins of the selected structures S5-S7 involved in this IIDA reaction are shown in Figure 6, while the complete BET study is gathered in Table S7 in the Supplementary Materials.

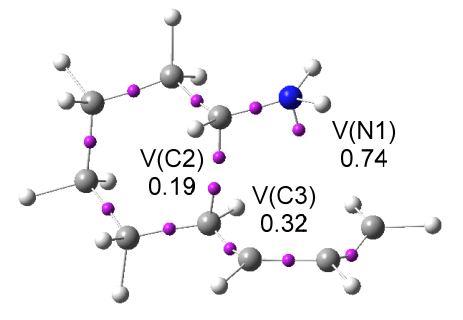

S5

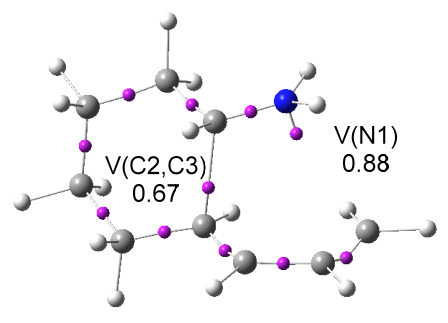

56

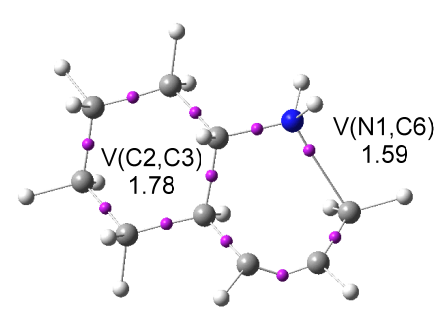

S7

Figure 6. ELF basin attractor positions, with the most significant valence basin populations, in average number of e, of the structures S5-S6 involved in the formation of the new N1-C6 and C2-C3 single bonds along the re/exo reaction path of the IIDA reaction of dieniminium $\mathbf{1 6}$.

The topology of the ELF of structure S5, d(N1-C6) $=2.840$ and $d(C 2-C 3)=2.055 \AA$, shows the presence of a $\mathrm{V}(\mathrm{N} 1)$ monosynaptic basin, integrating $0.74 \mathrm{e}$, and two monosynaptic basins, $\mathrm{V}(\mathrm{C} 2)$ and $\mathrm{V}(\mathrm{C} 3)$, integrating 0.19 and $0.32 \mathrm{e}$, respectively. These monosynaptic basins, which are not present in dieniminium 16, are created along the reaction path. While the $\mathrm{V}(\mathrm{N} 1)$ monosynaptic basin, resulting of the strong depopulation of the N1-C2 bonding region and the intramolecular GEDT taking place in this IIDA reaction, is demanded for the formation of the $\mathrm{N} 1-\mathrm{C} 6$ single bond, the $\mathrm{V}(\mathrm{C} 2)$ and $\mathrm{V}(\mathrm{C} 3)$ monosynaptic basins are needed for the subsequent formation of the first $\mathrm{C} 2-\mathrm{C} 3$ single bond. At S5, the GEDT is $0.40 \mathrm{e}$.

At structure S6, $\mathrm{d}(\mathrm{N} 1-\mathrm{C} 6)=2.822$ and $\mathrm{d}(\mathrm{C} 2-\mathrm{C} 3)=2.005 \AA$, while the two $\mathrm{V}(\mathrm{C} 2)$ and $\mathrm{V}(\mathrm{C} 3)$ monosynaptic basins present at structure $\mathrm{S} 5$ disappeared, a new $\mathrm{V}(\mathrm{C} 2, \mathrm{C} 3)$ disynaptic basin was created with an initial population of 0.67 e (see Figure 6). These relevant topological changes indicate that the formation of the first $\mathrm{C} 2-\mathrm{C} 3$ single bond starts at a C-C distance of $2.01 \AA$ by the merger of the non-bonding electron densities of the pseudoradical $\mathrm{C} 2$ and $\mathrm{C} 3$ centers present at structure $\mathrm{S} 5$ [8]. The V(N1) monosynaptic basin present at $\mathbf{S} 5$ reached a population of $0.88 \mathrm{e}$. At $\mathbf{S 6}$, the GEDT is $0.45 \mathrm{e}$.

Finally, at structure S7, d(N1-C6) $=2.115$ and $d(C 2-C 3)=1.580 \AA$, while the V(N1) monosynaptic basin present at structure $\mathbf{S 6}$ disappeared, a new $\mathrm{V}(\mathrm{N} 1, \mathrm{C} 6)$ disynaptic basin was created with an initial population of $1.59 \mathrm{e}$ (see Figure 6). These relevant topological changes indicate that the formation of the second N1-C6 single bond starts at a N-C distance of $2.11 \AA$, mainly by donation of the non-bonding electron density of the N1 nitrogen to the C6 carbon. At the point of the IRC before to the structure S6, the V(N1) monosynaptic basin present at $\mathbf{S} 5$ reached a population of $1.59 \mathrm{e}$. At $\mathbf{S 7}$, the GEDT is $0.65 \mathrm{e}$.

At structure $\mathbf{S 7}$, the $\mathrm{V}(\mathrm{C} 2, \mathrm{C} 3)$ disynaptic basin created at $\mathbf{S 6}$ reached an electron density of $1.78 \mathrm{e}$, a value that represents the $93 \%$ of the electron density of the C2-C3 single bond at quinolinium 17 . This behavior indicates that this IIDA reaction takes place through a non-concerted two-stage one-step mechanism [67].

3.3.2. ELF and AIM Comparative Topological Analysis of the Most Favorable TSs Involved in the Inter and Intra I-DA Reactions

Finally, an ELF and AIM comparative topological analysis of the electronic structures of the most favorable endo TSs involved in the I-DA reaction of butadiene $\mathbf{1}$ with ethaniminium 18, via TS1-n, and the IIDA reaction of dieniminium 16, via TS3-sn, was 
performed in order to establish the similarity in both inter and intramolecular I-DA reactions. The attractor positions of the ELF basins of TS1-n, and TS3-sn, together with the population of the more relevant valence basins and the presentations of the contour line maps of Laplacian $\nabla^{2}(r)$ of the electron density, together with the position of the critical points (CPs) associated with the $\mathrm{C} 2-\mathrm{C} 3$ and $\mathrm{N} 1-\mathrm{C} 6$ molecular regions, are shown in Figure 7, while the calculated AIM parameters of $\mathbf{C P 1}$ and $\mathbf{C P 2}$ are given in Table 6.

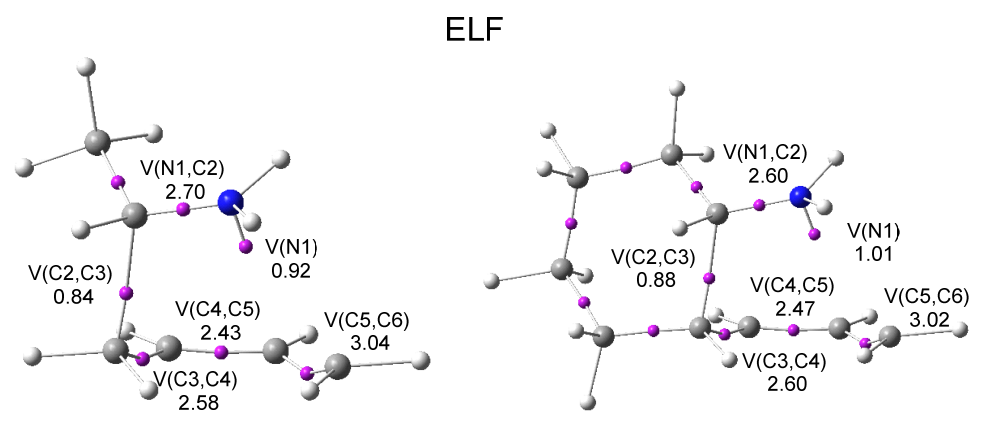

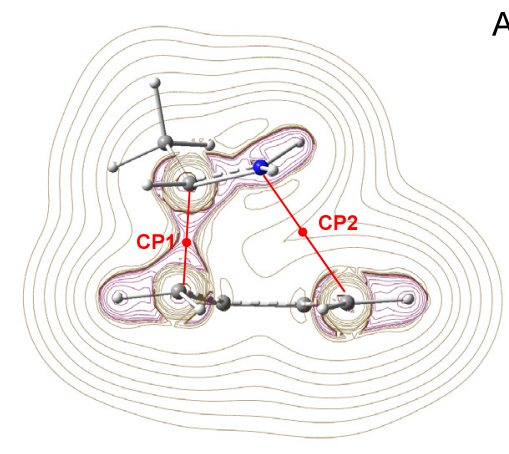

TS1-n

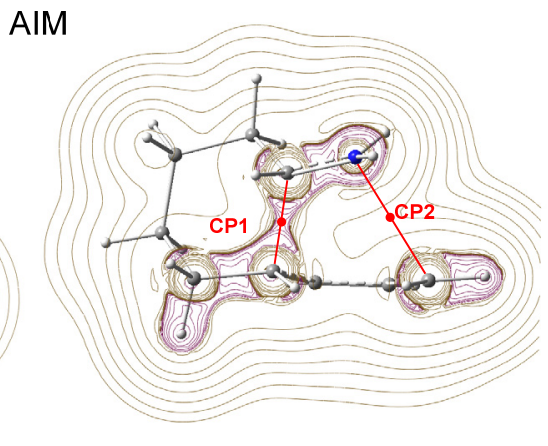

TS3-sn

Figure 7. (i) ELF basin attractor positions together with the most relevant valence basin populations at TS1-n and TS3-sn, and (ii) representations of the contour line maps of Laplacian $\nabla^{2}(\mathrm{r})$ of the electron density at TS1-n and TS3-sn. The CP1 and CP2 critical points are marked in the representations.

Table 6. AIM parameters, in au, of $(3,-1)$ CPs at TS1-n and TS3-sn in the regions associated with the $\mathrm{C}-\mathrm{C}$ and $\mathrm{C}-\mathrm{N}$ single bond formation.

\begin{tabular}{ccccc}
\hline & \multicolumn{2}{c}{ TS1-n } & \multicolumn{2}{c}{ TS3-sn } \\
\hline & CP1 & CP2 & CP1 & CP2 \\
\hline Density $\rho(r)$ & 0.0989 & 0.0142 & 0.1001 & 0.0176 \\
Laplacian $\nabla^{2}(r)$ & -0.0247 & 0.0364 & -0.0274 & 0.0444 \\
$G(r)$ & 0.0331 & 0.0079 & 0.0331 & 0.0099 \\
V(r) & -0.0723 & -0.0067 & -0.0732 & -0.0087 \\
$|\mathrm{~V}(\mathrm{r})| / \mathrm{G}(\mathrm{r})$ & 2.1843 & 0.8481 & 2.2115 & 0.8788 \\
$\mathrm{H}(\mathrm{r})$ & 0.0392 & 0.0012 & 0.0400 & 0.0012 \\
\hline
\end{tabular}

A comparison of the ELF of TS1-n, and TS3-sn shows that they have a great similarity. The iminium N1-C2 framework at both TSs is characterized by the presence of a V(N1-C2) disynaptic basin, integrating 2.70 and 2.60 e, respectively, and one $\mathrm{V}(\mathrm{N} 1)$ monosynaptic basin integrating 0.92 and $1.01 \mathrm{e}$. On the other hand, the butadiene moiety is characterized by the presence of three disynaptic basins, $\mathrm{V}(\mathrm{C} 3, \mathrm{C} 4), \mathrm{V}(\mathrm{C} 4, \mathrm{C} 5)$, and $\mathrm{V}(\mathrm{C} 5, \mathrm{C} 7)$, integrating a total of 8.05 and 8.09 e. In addition, the two TSs show the presence of a $\mathrm{V}(\mathrm{C} 2, \mathrm{C} 3)$ disynaptic basin, integrating 0.84 and $0.88 \mathrm{e}$.

While the presence of the $\mathrm{V}(\mathrm{C} 2, \mathrm{C} 3)$ disynaptic basins at the two TSs indicates that the formation of the first $\mathrm{C} 2-\mathrm{C} 3$ single bond has begun at these TSs, the presence of the 
$\mathrm{V}(\mathrm{N} 1)$ monosynaptic basins, which were created along the reaction paths, indicates that the formation of the second $\mathrm{N} 1-\mathrm{C} 2$ single bond has not yet begun.

Finally, the nature of the $\mathrm{C} 2-\mathrm{C} 3$ and N1-C6 electronic interactions at TS1-n and TS3-sn was studied by a AIM topological analysis of the electron density $\rho$ at the $(3,-1)$ $\mathrm{CPs}$ associated with the $\mathrm{C} 2-\mathrm{C} 3$ and $\mathrm{N} 1-\mathrm{C} 6$ molecular regions. The contour line maps of Laplacian $\nabla^{2}(r)$ of the electron density are shown in Figure 7, while the calculated AIM parameters are given in Table 6.

At TS1-n, CP1 shows a negative Laplacian $\nabla^{2}(\mathrm{r})$ value, -0.0247 , with an electron density of 0.0989 , while CP2 shows a positive Laplacian $\nabla^{2}(\mathrm{r})$ value, 0.0364 , with a low electron density of 0.0142. Similarly, at TS3-sn, CP1 shows a negative Laplacian $\nabla^{2}(\mathrm{r})$ value, -0.0274 , with an electron density of 0.1001 , while CP2 shows a positive Laplacian $\nabla^{2}(\mathrm{r})$ value, 0.0444 , with a low electron density of 0.0176 . Thus, while at the two TSs, the negative Laplacian $\nabla^{2}(\mathrm{r})$ values of $\mathbf{C P 1}$ indicate the presence of covalent interactions, the positive Laplacian $\nabla^{2}(\mathrm{r})$ values of $\mathbf{C P 2}$ indicate the absence of any covalent interactions.

Espinosa [68] proposed a useful criterion to characterize interactions at the CPs, the ratio of potential kinetic energy electron density, $|\mathrm{V}(\mathrm{r})| / \mathrm{G}(\mathrm{r})$. For ionic, non-covalent, and hydrogen bonding interactions, $|\mathrm{V}(\mathrm{r})| / \mathrm{G}(\mathrm{r})<1$. Non-covalent interactions with somewhat covalent character are characterized by $1<|\mathrm{V}(\mathrm{r})| / \mathrm{G}(\mathrm{r})<2$, while covalent interactions show $|V(r)| / G(r)>2$. The calculated values of $|V(r)| / G(r)$ at the selected CPs associated with TS1-n and TS3-sn are given in Table 6. The $|\mathrm{V}(\mathrm{r})| / \mathrm{G}(\mathrm{r})$ values of CP1 at these TSs, between 2.18 and $2.21 \mathrm{au}$, indicate that they are associated with covalent interactions, while the $|\mathrm{V}(\mathrm{r})| / \mathrm{G}(\mathrm{r})$ values of $\mathbf{C P 2}$, between 0.85 and $0.88 \mathrm{au}$, indicate that they are associated with non-covalent interactions.

The present ELF and AIM topological analysis of the electron density of TS1-n and TS3-sn shows that there is a complete similitude between the electronic structure of both TSs, in complete agreement with the similar geometries and GEDT of them.

\section{Conclusions}

The IIDA reactions of dieniminiums $\mathbf{1 2}$ and $\mathbf{1 6}$ were studied within the MEDT at the DFT $\omega$ B97XD/6-311G(d,p) computational level. The ELF topological analysis of dieniminiums 12 and 16 showed that the electronic structure of these species can be seen as the sum of those of butadiene 1 and that of ethaniminium 18 joined by the tetramethylene chain. Analysis of the electrophilicity $\omega$ index of dieniminiums 12 and 16 pointed to the superelectrophilic character of these species. On the other hand, analysis of the Parr functions allowed characterization of the most electrophilic center, the iminium C2 carbon, and the most nucleophilic centers, the $\mathrm{C} 3$ and $\mathrm{C} 6$ carbons of the butadiene moiety, of dieniminium 16.

The IIDA reaction of dieniminium 12 takes place via a one-step mechanism. The activation enthalpy associated with this IIDA reaction, $8.7 \mathrm{kcal} \cdot \mathrm{mol}^{-1}$, is closer to that of the I-DA reaction between butadiene 1 and ethaniminium $18,9.3 \mathrm{kcal} \cdot \mathrm{mol}^{-1}$. However, while the intermolecular I-DA reaction has an activation Gibbs free energy of $25.3 \mathrm{kcal} \cdot \mathrm{mol}^{-1}$, this IIDA reaction presents an activation Gibbs free energy of only $12.6 \mathrm{kcal} \cdot \mathrm{mol}^{-1}$ as a consequence of the very low activation entropy associated with this intramolecular process, $-14.3 \mathrm{cal} \cdot \mathrm{mol}^{-1} \cdot \mathrm{K}$. The strong exergonic character of the IIDA reaction, higher than $20.5 \mathrm{kcal} \cdot \mathrm{mol}^{-1}$, makes the reaction irreversible.

Unlike I-DA reactions, which are low endo stereoselective [13], these IIDA reactions present a total re/exo and si/endo diastereoselectivity, which is controlled for the most favorable chair conformations adopted by the tetramethylene chain along the re and si intramolecular approaches. Such as in the I-DA reactions, the IIDA reaction of dieniminium 12 presents a very high intramolecular GEDT, being classified as FEDF.

ELF topological analysis of the single bond formation along the IIDA reaction of dieniminium 16 indicated that it takes place through a non-concerted two-stage one-step mechanism. While formation of the first $\mathrm{C} 2-\mathrm{C} 3$ single bond begins by the merger of the non-bonding electron densities of the two pseudoradical $\mathrm{C} 2$ and $\mathrm{C} 3$ centers created along 
the reaction path, the formation of the second $\mathrm{N} 1-\mathrm{C} 6$ single bond begins mainly by the donation of the non-bonding electron density of the N1 nitrogen on the C6 carbon. Finally, ELF and AIM topological analyses of the more favorable TS associated with the I-DA reaction between butadiene 1 and ethaniminium 18 and that associated with the IIDA reaction of dieniminium $\mathbf{1 6}$ showed the great similarity of the two TSs.

The present MEDT study raises an interesting issue. The high GEDT taking place at the I-DA reactions is rationalized by the high $\Delta \mu$ values of the electronic chemical potentials $\mu$ of the two interacting species. Similar to iminiums, dieniminiums presented very low electronic chemical potentials $\mu$ values, lesser than $-7.0 \mathrm{eV}$, suggesting a high GEDT at the corresponding TSs. The Sanderson's Electronegativity Equalization Principle $[69,70]$ establishes that, along a polar interaction, the GEDT fluxes from the species with the high electronic chemical potentials $\mu$ to those with a low value. However, what happens in the intramolecular processes for which the electrophilic $P_{k}^{+}$and nucleophilic $P_{k}^{-}$Parr functions recognize two distinguishable frameworks with electrophilic/nucleophilic properties similar to those present in intermolecular processes?

The answer to this question is that a regional descriptor for the electronic chemical potential $\mu_{\mathrm{f}}$ for the different fragments of a molecule able to explain the GEDT taking place in intramolecular processes should be established.

Supplementary Materials: The following are available online at https: / www.mdpi.com/article / 10.3390 / chemistry3030061/s1. Estimation of the electrophilic $\omega$ indices from the LUMO energies. Figure with the ELF basin attractor positions of the experimental dieniminium 12e. Figure with the geometries of the TSs involved in the IIDA reaction of dieniminium 16. Table with the B3LYP/6$311 \mathrm{G}(\mathrm{d}, \mathrm{p})$ thermodynamic data of the stationary points involved in the N-DA reaction of butadiene 1 and ethylene 2, and in the IMDA reaction of $(E)$-deca-1,3,9-triene 10. Table with the total energies and the thermodynamic data of the stationary points involved in the I-DA reaction of butadiene 1 with ethaniminium 18. Table with the $\omega \mathrm{B} 97 \mathrm{XD} / 6-311 \mathrm{G}(\mathrm{d}, \mathrm{p})$ total energies of the stationary points involved in the IIDA reactions of dieniminiums 12 and 16. Table with the thermodynamic data of the stationary points involved in the in the IIDA reaction of dieniminium 12. Table with the ELF valence basin populations and distances of the N1-C6 and C2-C3 forming bonds of the structures of the IRC associated with the IIDA reaction of dieniminium 16, defining the nine phases of the BET.

Author Contributions: L.R.D. headed the subject, wrote the manuscript, and performed calculations; M.R.-G. performed calculations and wrote the manuscript, and M.J.A. performed calculations and wrote the manuscript. All authors have read and agreed to the published version of the manuscript.

Funding: This research received funding from the Ministry of Economy and Competitiveness (MINECO) of the Spanish Government, project PID2019-110776GB00 (AEI/FEDER, UE), and the European Union's Horizon 2020 research and innovation program under the Marie Skłodowska-Curie grant agreement No. 84618.

Data Availability Statement: Data is contained within the article or supplementary material, or can be made available upon written request to the corresponding author.

Acknowledgments: L.R.D. and M.J.A. acknowledge the Ministry of Economy and Competitiveness (MINECO) of the Spanish Government, and M.R.-G. acknowledge the European Commission for financial support.

Conflicts of Interest: The authors declare no conflict of interest.

Sample Availability: Samples of the compounds are not available from the authors.

\section{References}

1. Diels, O.; Alder, K. Synthesen in der hydroaromatischen Reihe. Justus Liebigs Ann. Chem. 1928, 460, 98-122. [CrossRef]

2. Carruthers, W. Some Modern Methods of Organic Synthesis, 2nd ed.; Cambridge University Press: Cambridge, UK, 1978.

3. Carruthers, W. Cycloaddition Reactions in Organic Synthesis; Pergamon: Oxford, UK, 1990.

4. Woodward, R.B.; Hoffmann, R. The Conservation of Orbital Symmetry. Angew. Chem. Int. Ed. Engl. 1969, 8, 781-853. [CrossRef]

5. Houk, K.N.; Gonzalez, J.; Li, Y. Pericyclic Reaction Transition States: Passions and Punctilios, 1935-1995. Acc. Chem. Res. 1995, 28, 81-90. [CrossRef]

6. Rowley, D.; Steiner, H. Kinetics of diene reactions at high temperatures. Discuss. Faraday Soc. 1951, 10, 198-213. [CrossRef] 
7. Goldstein, E.; Beno, B.; Houk, K.N. Density Functional Theory Prediction of the Relative Energies and Isotope Effects for the Concerted and Stepwise Mechanisms of the Diels-Alder Reaction of Butadiene and Ethylene. J. Am. Chem. Soc. 1996, 118, 6036-6043. [CrossRef]

8. Domingo, L.R. A new C-C bond formation model based on the quantum chemical topology of electron density. RSC Adv. 2014, 4, 32415-32428. [CrossRef]

9. Domingo, L.R.; Arnó, M.; Andrés, J. Influence of Reactant Polarity on the Course of the Inverse-Electron-Demand Diels-Alder Reaction. A DFT Study of Regio- and Stereoselectivity, Presence of Lewis Acid Catalyst, and Inclusion of Solvent Effects in the Reaction between Nitroethene and Substituted Ethenes. J. Org. Chem. 1999, 64, 5867-5875.

10. Domingo, L.R.; Aurell, M.J.; Perez, P.; Contreras, R. Origin of the synchronicity on the transition structures of polar Diels-Alder reactions. Are these reactions [4+2] processes? J. Org. Chem. 2003, 68, 3884-3890. [CrossRef] [PubMed]

11. Domingo, L.R.; Sáez, J.A. Understanding the mechanism of polar Diels-Alder reactions. Org. Biomol. Chem. 2009, 7, 3576-3583. [CrossRef]

12. Domingo, L.R. Molecular electron density theory: A modern view of reactivity in organic chemistry. Molecules 2016, 21, 1319. [CrossRef]

13. Domingo, L.R.; Ríos-Gutiérrez, M.; Aurell, M.J. Unveiling the Ionic Diels-Alder Reactions within the Molecular Electron Density Theory. Molecules 2021, 26, 3638. [CrossRef] [PubMed]

14. Sustmann, R.; Trill, H. Substituent Effects in 1,3-Dipolar Cycloadditions of Phenyl Azide. Angew. Chem. Int. Ed. Engl. 1972, 11, 838-840. [CrossRef]

15. Fukui, K. Molecular Orbitals in Chemistry, Physics, and Biology; Löwdin, P.-O., Pullman, B., Eds.; Academic Press: New York, NY, USA, 1964.

16. Houk, K.N.; Sims, J.; Watts, C.R.; Luskus, L.J. Origin of reactivity, regioselectivity, and periselectivity in 1,3-dipolar cycloadditions. J. Am. Chem. Soc. 1973, 95, 7301-7315. [CrossRef]

17. Domingo, L.R.; Ríos-Gutiérrez, M.; Silvi, B.; Pérez, P. The Mysticism of Pericyclic Reactions. A Contemporary Rationalisation of Organic Reactivity Based on the Electron Density Analysis. Eur. J. Org. Chem. 2018, 2018, 1107-1120. [CrossRef]

18. Kiselev, V.D.; Konovalov, A.I. Factors that determine the reactivity of reactants in normal and catalysed Diels-Alder reactions. Russ. Chem. Rev. 1989, 58, 230-249. [CrossRef]

19. Anh, N.T.; Maurel, F. Use and misuse of frontier orbital theory. New J. Chem. 1997, 21, 861-871.

20. Spino, C.; Rezaei, H.; Dory, Y.L. Characteristics of the Two Frontier Orbital Interactions in the Diels-Alder Cycloaddition. J. Org. Chem. 2004, 69, 757-764. [CrossRef]

21. Domingo, L.R. Why do electron-deficient dienes react rapidly in Diels-Alder reactions with electron-deficient ethylenes? A density functional theory analysis. Eur. J. Org. Chem. 2004, 2004, 4788-4793. [CrossRef]

22. Domingo, L.R.; Ríos-Gutiérrez, M.; Pérez, P. A Molecular Electron Density Theory Study of the Reactivity of Tetrazines in Aza-Diels-Alder Reactions. RSC Adv. 2020, 10, 15394-15405. [CrossRef]

23. Domingo, L.R.; Kula, K.; Ríos-Gutiérrez, M. Unveiling the Reactivity of Cyclic Azomethine Ylides in [3 + 2] Cycloaddition Reactions within the Molecular Electron Density Theory. Eur. J. Org. Chem. 2020, 2020, 5938-5948. [CrossRef]

24. Soto-Delgado, J.; Aizman, A.; Contreras, R.; Domingo, L.R. On the Catalytic Effect of Water in the Intramolecular Diels-Alder Reactions of Quinone System. A Theoretical Study. Molecules 2012, 17, 13687-13703. [CrossRef] [PubMed]

25. Soto-Delgado, J.; Aizman, A.; Domingo, L.R.; Contreras, R. Invariance of electrophilicity of independent fragments. Application to intramolecular Diels-Alder reactions. Chem. Phys. Lett. 2010, 499, 272-277. [CrossRef]

26. Soto-Delgado, J.; Domingo, L.R.; Contreras, R. Quantitative characterization of group electrophilicity and nucleophilicity for intramolecular Diels-Alder reactions. Org. Biomol. Chem. 2010, 8, 3678-3683. [CrossRef]

27. Liang, Y.; Jiang, X.; Yu, Z.-X. Mechanisms of Cascade Reactions in the Syntheses of Camptothecin-Family Alkaloids: Intramolecular [4+ + 2]. Reactions of N-Arylimidates and Alkynes. Org. Lett. 2009, 22, 5303-5305. [CrossRef]

28. Zhang, Y.; Zhu, Y.; Zheng, L.; Zhuo, 1.-G.; Dang, Q.; Yu, Z.-X.; Bai, X. On-Demand Selection of the Reaction Path from Imino Diels-Alder to Ene-Type Cyclization: Synthesis of Epiminopyrimido[4,5-b]azepines. Eur. J. Org. Chem. 2014, 660, 660-669. [CrossRef]

29. Grieco, P.A.; Parker, D.T. Octahydroquinoline Synthesis via Immonium Ion Based Diels-Alder Chemistry: Synthesis of (-)-8aEpipumiliotoxin C. J. Org. Chem. 1988, 53, 3658-3662. [CrossRef]

30. Becke, A.D. Density-functional thermochemistry. The role of exact Exchange. J. Chem. Phys. 1993, 98, 5648-5652. [CrossRef]

31. Lee, C.; Yang, W.; Parr, R.G. Phys. Development of the Colle-Salvetti correlation-energy formula into a functional of the electron density. Phys. Rev. B 1988, 37, 785-789. [CrossRef] [PubMed]

32. Chai, J.-D.; Head-Gordon, M. Long-range corrected hybrid density functionals with damped atom-atom dispersion corrections. Phys. Chem. Chem. Phys. 2008, 10, 6615-6620. [CrossRef]

33. Hehre, M.J.; Radom, L.; Schleyer, P.V.R.; Pople, J. Ab initio Molecular Orbital Theory; Wiley: New York, NY, USA, 1986.

34. Schlegel, H.B. Optimization of equilibrium geometries and transition structures. J. Comput. Chem. 1982, 3, 214-218. [CrossRef]

35. Schlegel, H.B. Modern Electronic Structure Theory; Yarkony, D.R., Ed.; World Scientific Publishing: Singapore, 1994.

36. Fukui, K. Formulation of the reaction coordinate. J. Phys. Chem. 1970, 74, 4161-4163. [CrossRef] 
37. González, C.; Schlegel, H.B. Reaction path following in mass-weighted internal coordinates. J. Phys. Chem. 1990, 94, 5523-5527. [CrossRef]

38. González, C.; Schlegel, H.B. Improved algorithms for reaction path following: Higher-order implicit algorithms. J. Chem. Phys. 1991, 95, 5853-5860. [CrossRef]

39. Tomasi, J.; Persico, M. Molecular interactions in solution: And overview of methods based on continuous distributions of the solvent. Chem. Rev. 1994, 94, 2027-2094. [CrossRef]

40. Simkin, B.Y.; Sheikhet, I.I. Quantum Chemical and Statistical Theory of Solutions-Computational Approach; Ellis Horwood: London, UK, 1995.

41. Cossi, M.; Barone, V.; Cammi, R.; Tomasi, J. Ab initio study of solvated molecules: A new implementation of the polarizable continuum model. Chem. Phys. Lett. 1996, 255, 327-335. [CrossRef]

42. Cances, E.; Mennucci, B.; Tomasi, J. A new integral equation formalism for the polarizable continuum model: Theoretical background and applications to isotropic and anisotropic dielectrics. J. Chem. Phys. 1997, 107, 3032-3041. [CrossRef]

43. Barone, V.; Cossi, M.; Tomasi, J. Geometry optimization of molecular structures in solution by the polarizable continuum model. J. Comput. Chem. 1998, 19, 404-417. [CrossRef]

44. Reed, A.E.; Weinstock, R.B.; Weinhold, F. Natural population analysis. J. Chem. Phys. 1985, 83, 735-746. [CrossRef]

45. Reed, A.E.; Curtiss, L.A.; Weinhold, F. Intermolecular interactions from a natural bond orbital, donor-acceptor viewpoint. Chem. Rev. 1988, 88, 899-926. [CrossRef]

46. Parr, R.G.; Yang, W. Density Functional Theory of Atoms and Molecules; Oxford University Press: New York, NY, USA, 1989.

47. Domingo, L.R.; Ríos-Gutiérrez, M.; Pérez, P. Applications of the conceptual density functional indices to organic chemistry reactivity. Molecules 2016, 21, 748. [CrossRef] [PubMed]

48. Frisch, M.J.; Trucks, G.W.; Schlegel, H.B.; Scuseria, G.E.; Robb, M.A.; Cheeseman, J.R.; Scalmani, G.; Barone, V.; Petersson, G.A.; Nakatsuji, H.; et al. Gaussian 16, Revision A.03; Gaussian, Inc.: Wallingford, CT, USA, 2016.

49. Becke, A.D.; Edgecombe, K.E. A simple measure of electron localization in atomic and molecular-systems. J. Chem. Phys. 1990, 92, 5397-5403. [CrossRef]

50. Noury, S.; Krokidis, X.; Fuster, F.; Silvi, B. Computational tools for the electron localization function topological analysis. Comput. Chem. 1999, 23, 597-604. [CrossRef]

51. Bader, R.F.W.; Tang, Y.H.; Tal, Y.; Biegler-König, F.W. Properties of atoms and bonds in hydrocarbon molecules. J. Am. Chem. Soc. 1982, 104, 946-952. [CrossRef]

52. Lu, T.; Chen, F. Multiwfn: A multifunctional wavefunction analyzer. J. Comp. Chem. 2012, 33, 580-592. [CrossRef] [PubMed]

53. GaussView, Version 6.0; Dennington, R.; Keith, T.A.; Millam, J.M. (Eds.) Semichem Inc.: Shawnee Mission, KS, USA, 2016.

54. Ahrens, J.; Geveci, B.; Law, C. ParaView: An End-User Tool for Large Data Visualization. In Visualization Handbook; Elsevier: Amsterdam, The Netherlands, 2005.

55. Ayachit, U. The ParaView Guide: A Parallel Visualization Application; Kitware: New York, NY, USA, 2015; ISBN 978-1930934306.

56. Silvi, B.; Savin, A. Classification of chemical bonds based on topological analysis of electron localization functions. Nature 1994, 371, 683-686. [CrossRef]

57. Pérez, P.; Domingo, L.R.; Aizman, A.; Contreras, R. The Electrophilicity Index in Organic Chemistry. In Theoretical Aspects of Chemical Reactivity; Toro-Labbe, A., Ed.; Elsevier: Amsterdam, The Netherlands, 2007; Volume 9, pp. $139-201$.

58. Domingo, L.R.; Pérez, P. The Nucleophilicity N Index in Organic Chemistry. Org. Biomol. Chem. 2011, 9, 7168-7175. [CrossRef]

59. Parr, R.G.; Pearson, R.G. Absolute hardness: Companion parameter to absolute electronegativity. J. Am. Chem. Soc. 1983, 105, 7512-7516. [CrossRef]

60. Parr, R.G.; Szentpaly, L.v.; Liu, S. Electrophilicity index. J. Am. Chem. Soc. 1999, 121, 1922-1924. [CrossRef]

61. Domingo, L.R.; Pérez, P. The Lithium Cation Catalysed Benzene Diels-Alder reaction. Insights on the Molecular Mechanism within the Molecular Electron Density Theory. J. Org. Chem. 2020, 85, 13121-13132. [CrossRef] [PubMed]

62. Domingo, L.R.; Chamorro, E.; Pérez, P. Understanding the reactivity of captodative ethylenes in polar cycloaddition reactions. A theoretical study. J. Org. Chem. 2008, 73, 4615-4624. [CrossRef] [PubMed]

63. Aurell, M.J.; Domingo, L.R.; Perez, P.; Contreras, R. A theoretical study on the regioselectivity of 1,3-dipolar cycloadditions using DFT-based reactivity indexes. Tetrahedron 2004, 60, 11503-11509. [CrossRef]

64. Domingo, L.R.; Perez, P.; Sáez, J.A. Understanding the local reactivity in polar organic reactions through electrophilic and nucleophilic Parr functions. RSC Adv. 2013, 31486-31494. [CrossRef]

65. Domingo, L.R.; Rios-Gutierrez, M.; Ndassa, I.A.I.M.; Nouhou, C.N.; Mbadcam, J.K. Molecular Electron Density Theory Study of Fused Regioselectivity in the Intramolecular [3+2] Cycloaddition Reaction of Nitrones. Chem. Select. 2018, 3, 5412-5420. [CrossRef]

66. Krokidis, X.; Noury, S.; Silvi, B. Characterization of Elementary Chemical Processes by Catastrophe Theory. J. Phys. Chem. A 1997, 101, 7277-7282. [CrossRef]

67. Domingo, L.R.; Sáez, J.A.; Zaragozá, R.J.; Arnó, M. Understanding the Participation of Quadricyclane as Nucleophile in Polar [2 $\sigma$ $+2 \sigma+2 \pi$ ] Cycloadditions toward Electrophilic $\pi$ Molecules. J. Org. Chem. 2008, 73, 8791-8799. [CrossRef] [PubMed]

68. Espinosa, E.; Alkorta, I.; Elguero, J.; Molins, E. From weak to strong interactions: A comprehensive analysis of the topological

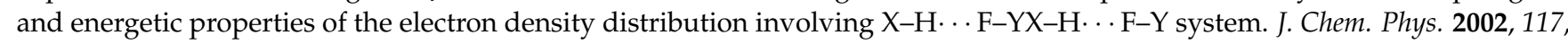
5529-5542. [CrossRef] 
69. Sanderson, R.T. Partial charges on atoms in organic compounds. Science 1955, 121, 207-208. [CrossRef] [PubMed] 70. Sanderson, R.T. Chemical Bonds and Bond Energy, 2nd ed.; Academic Press: New York, NY, USA, 1976. 